\title{
THIN FILM OPTICAL PARAMETERS DETERMINATION BY THE DYNAMICAL MODELLING AND STOCHASTIC OPTIMIZATION METHOD
}

We report on a new method of experimental data processing to obtain optical parameters of thin films. Dynamical modelling of the spectral reflectance can be performed interactively in a graphical environment by the genetic search in wide interval of parameter space and then refined by the genetic algorithm method, by the Nelder-Mead downhill simplex method or Marquardt-Levenberg method. Optical parameters of hydrogenated amorphous silicon (a-Si:H) thin film used in solar cell technology are determined by this new method. The spectral reflectance is a function of optical properties and the thickness of the film. Optical parameters found by our approach do not depend on the initial spectral reflectance estimation.

\section{Introduction}

The theoretical and experimental studies on the optical behaviour of thin films deal primarily with optical reflection, transmission and absorption properties, and their relation to the optical constants of films. The theoretical reflectance $R_{t}$ can be calculated from the optical properties and thickness $d$ of the film. Normal incidence reflectometry is often used for several reasons: good spatial resolution, high throughput and accuracy. Optical properties of any material can be described by the complex index of refraction, $N=n-i k$, where $n$ is the refractive index and $k$ is the extinction coefficient. Both $n$ and $k$ depend on the wavelength of light $\lambda$. In many thin-film applications, namely in solar cell technologies, the optical constants $n$ and $k$ are strongly correlated to the deposition process conditions and their broadband determination is needed to design the solar cell structures. Experimental and theoretical reflectance can be matched by fitting for the film thickness and optical properties. The optimization problem is formulated as

$$
\min _{3}\left\{\sum\left[R_{o}(\lambda)-R_{t}(n, k, \lambda, d)\right]^{2}\right\}
$$

where $R_{o}$ denotes measured reflectance values. The optimization issue is to find the system of parameters that satisfy the desired optical specifications. Numerical methods are flexible and can handle complicated problems. The numerical computations are based on merit functions that can be defined according to quantities of interest. Considering the computational cost involved, and furthermore the constraints to calculate the gradient information for this specific optical thin-film model, algorithms that can substantially reduce computation without using derivatives are preferable. In our approach the dynamical modelling and stochastic optimization approach (DMSO) is used.

\section{Dynamical modelling and stochastic optimization method}

The spectral reflectance (SR) of a thin quasi-ideal single homogeneous isotropic film on a thick partly absorbing substrate is given by

$$
R=\frac{A+B x+C x^{2}}{D+E x+F x^{2}} .
$$

In this equation $x=\exp (-\alpha d)$ is the absorbance, $\alpha=\left(4 \pi k_{1}\right) / \lambda$ is the absorption coefficient [1] and

$$
\begin{aligned}
& A=\left[\left(1-n_{1}\right)^{2}+k_{1}^{2}\right]\left[\left(n_{1}+n_{2}\right)^{2}+\left(k_{1}+k_{2}\right)^{2}\right] \\
& B=2\left[A^{\prime} \cos \varphi+B^{\prime} \sin \varphi\right] \\
& C=\left[\left(1+n_{1}\right)^{2}+k_{1}^{2}\right]\left[\left(n_{1}-n_{2}\right)^{2}+\left(k_{1}-k_{2}\right)^{2}\right] \\
& D=\left[\left(1+n_{1}\right)^{2}+k_{1}^{2}\right]\left[\left(n_{1}+n_{2}\right)^{2}+\left(k_{1}+k_{2}\right)^{2}\right] \\
& E=2\left[C^{\prime} \cos \varphi+D^{\prime} \sin \varphi\right] \\
& F=\left[\left(1-n_{1}\right)^{2}+k_{1}^{2}\right]\left[\left(n_{1}-n_{2}\right)^{2}+\left(k^{1}-k_{2}\right)^{2}\right] \\
& A^{\prime}=\left(1-n_{1}^{2}-k_{1}^{2}\right)\left(n_{1}^{2}-n_{2}^{2}+k_{1}^{2}-k_{2}^{2}\right)+4 k_{1}\left(n_{1} k_{2}-n_{2} k_{1}\right) \\
& B^{\prime}=2\left(1-n_{1}^{2}-k_{1}^{2}\right)\left(n_{1} k_{2}-n_{2} k_{1}\right)-2 k_{1}\left(n_{1}^{2}-n_{2}^{2}+k_{1}^{2}-k_{2}^{2}\right) \\
& C^{\prime}=\left(1-n_{1}^{2}-k_{1}^{2}\right)\left(n_{1}^{2}-n_{2}^{2}+k_{1}^{2}-k_{2}^{2}\right)-4 k_{1}\left(n_{i} k_{2}-n_{2} k_{1}\right) \\
& D^{\prime}=2\left(1-n_{1}^{2}-k_{1}^{2}\right)\left(n_{1} k_{2}-n_{2} k_{1}\right)+2 k_{1}\left(n_{1}^{2}-n_{2}^{2}+k_{1}^{2}-k_{2}^{2}\right) \\
& \varphi=\left(4 \pi n_{1} d\right) / \lambda
\end{aligned}
$$

In the DMSO method, the optical parameters are determined in several steps. In the first step we prepare an initial spectral reflectance model in graphical environment by interactive dynamical modification of the reflectance model parameters. Alternatively automatic stochastic search in wide interval of parameter hyperspace can be used to create an initial reflectance model. The stochastic optimization is based on the genetic algorithm (GA). The next step uses stochastic optimization refinement of result reflectance reached in the previous modelling by the Nelder-Mead or Marquardt-Levenberg optimization:

\footnotetext{
* S. Jurečka, J. Müllerová

Department of Engineering Fundamentals, Faculty of Electrical Engineering, kpt. Nálepku 1390, 03101 L. Mikuláš, Slovakia,

E-mail: jurecka@lm.utc.sk
} 


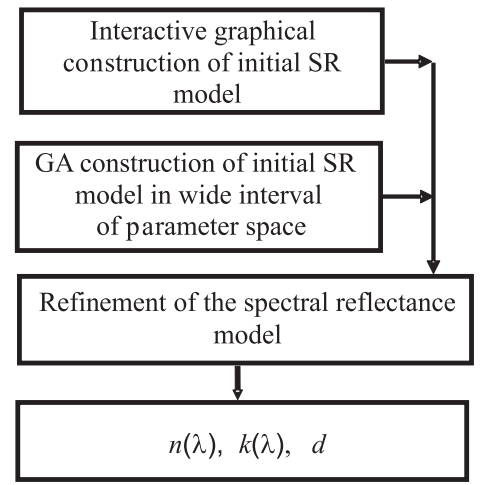

Fig. 1 Dynamical modelling method of the initial spectral reflectance model estimation

The step of dynamical modelling speeds up the calculations of the reflectance function parameters and enables constructing the theoretical reflectance model with comfort and high quality. Besides this, stochastic algorithms enable also comfortable incorporation of necessary physical constraints into the optimization method. We used genetic algorithm with binary representation of the theoretical reflectance model variables [2]. There are many different settings associated with predictions in optical parameters solutions. Most of these settings are maintained automatically using rules of the GA that create the best results. To speed up the achievement of the best solution we used visual construction of the spectral reflectance model. The values of the theoretical reflectance model parameters are dynamically modified and corresponding theoretical model is graphically displayed and compared with an experimental reflectance curve. This visual modelling step provides good estimation of the initial reflectance model and substantially reduces computation time and stochastic search of the model parameters values.

GA procedure refines the initial mathematical model by minimizing the differences between theoretical and experimental values. The initial reflectance estimation introduces a set of parameters based obviously on the properly selected dispersion model of the refractive index $n$ and the extinction coefficient $k$ [3,4], e.g.

$$
n=n\left(p_{1}, p_{2}, p_{3} \cdot p_{4}, \lambda\right), \quad k=k\left(p_{1}, p_{2}, p_{3} \cdot p_{4}, \lambda\right) .
$$

Therefore, the spectral reflectance model is represented by variables

$$
X=\left\{p_{1}, p_{2}, p_{3} \cdot p_{4}, \lambda\right\}
$$

A set of correction values $\Delta=\left\{\Delta p_{1}, \Delta p_{2}, \Delta p_{3} . \Delta p_{4}, \lambda\right]$ is used in each GA iteration step for setting new trial reflectance model variables $X^{\prime}=X-\Delta$. The implementation of the GA algorithm minimizes the differences (1) by stochastic exploring the correction parameters hyperspace in the interval $\langle-0.2 X, 0.2 X\rangle$ for each variable in the spectral reflectance function. Alternatively in case of the high quality of the initial spectral reflectance mathematical model was obtained in the first step of the DMSO method the conventional numerical optimization methods can be implemented (Marquardt-Levenberg or Nelder-Mead simplex methods):

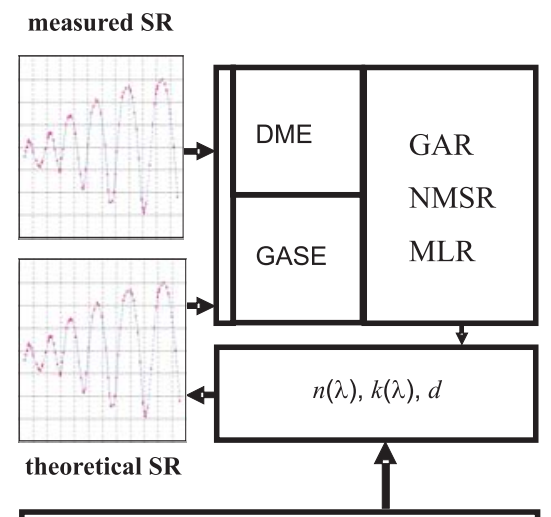

SR theoretical model dispersion relations

Fig. 2 DMSO method implementation

DME - Dynamical Modelling Initial SR Model Estimation

GASE - Genetic Algorithm Search SR Estimation

GAR - Genetic Algorithm method SR Refinement

NMSR - Nelder-Mead Simplex method SR Refinement

MLR - Marquardt-Levenberg method SR Refinement

\section{Experimental results}

The described method was used to extract the refractive indices of a series of undoped a-Si:H thin films with approximately the same thickness $\sim 400 \mathrm{~nm}$ (Table 1). The samples were deposited at the Delft University of Technology, the Netherlands, on Corning 1737 glass substrates by PECVD industrial deposition system [5,6] from hydrogen $\left(\mathrm{H}_{2}\right)$ to silane $\left(\mathrm{SiH}_{4}\right)$ plasma under varied $\mathrm{H}_{2} / \mathrm{SiH}_{4}$ gas flows (the dilution D).

Samples under study

Table 1

\begin{tabular}{|c|c|}
\hline dilution D & thickness [nm] \\
\hline 0 & 390 \\
\hline 10 & 394 \\
\hline 20 & 385 \\
\hline 30 & 388 \\
\hline 40 & 402 \\
\hline 50 & 397 \\
\hline
\end{tabular}

Optical properties of the dilution series are obviously affected by the changing structure [6]. The refractive indices for all samples under study were determined from the experimental spectral reflectance determined by the DMSO method (Fig. 3). At all wavelengths, we observe the decreases of the refractive index with increasing dilution For the sample prepared at $D=40$, approximately the same spectral refractive indices were revealed as for the 


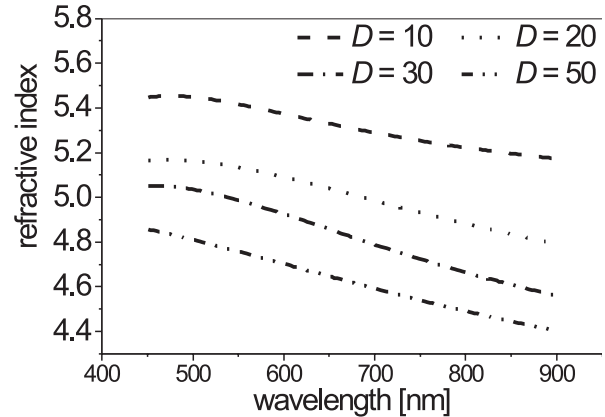

Fig. 3 Refractive indices of the a-Si:H thin film samples.

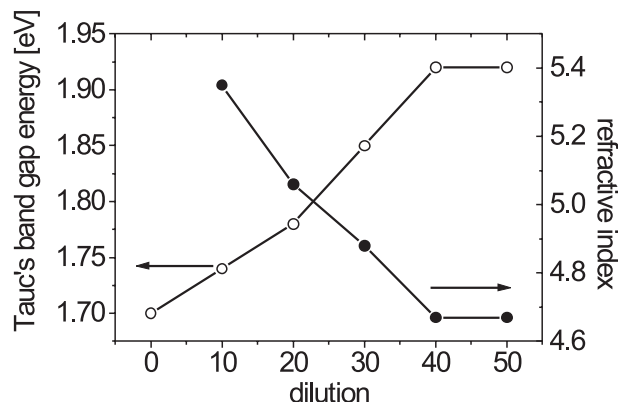

Fig. 4 Refractive index (at $633 \mathrm{~nm}$ ) and the Tauc's optical band.

sample at $D=50$, therefore this plot was not incorporated into the Fig. 3.

Fig. 4 illustrates the dilution-dependent refractive index at $633 \mathrm{~nm}$ and Tauc's optical band gap determined from the experimental spectral transmittance in the vicinity of the absorption edge [6]. The data for Tauc's optical band gap as one member of the set of dispersion model parameters acquired from the course of DMSO method are in good agreement with the values plotted in Fig. 4.

Thus, the DMSO method yields quickly and reliably information useful for all applications of the samples under study where optical properties matter. The observed decrease of refractive index may be probably due to the voids - sites with missing radicals of $\mathrm{SiH}_{4}-\mathrm{H}_{2}$ decomposition. The band gap appears to increase with increasing dilution. The increase of $E_{g}$ at the amorphous-crystalline boundary is due to the appearance of indirect gap electronic structure of the crystalline silicon.

\section{Conclusions}

The DMSO method was introduced for the thin film optical parameter determination. Experimental spectral reflectance data of a series of a-Si:H thin films were analysed. DMSO method succeeded in theoretical reflectance model refinement very good. It does not converge to a local optimum of the refined model. The combination of dynamical modelling and stochastic optimization of the initial mathematical model compared with the experimental data speeds up calculations and enables checking for meaningful optical parameters.

\section{Acknowledgements}

This work was supported in part by the Slovak Grant Agency under grants No. 2/4105/04 and No. 2/4100/04. Dr Nádaždy at the Institute of Physics, Slovak

Academy of Sciences, Bratislava, Slovakia, is acknowledged for the sample preparation.

\section{References}

[1] SWANEPOEL, R.: J. Phys. E, 34, 1214, 1983.

[2] GOLDBERG, D. E.: Genetic Algorithms in Search, Optimization and Machine Learning. Addisom-Wesley, 1989.

[3] FRANTA, D., OHLÍDAL, I., MUNZAR, D.: Acta Physica Slovaca, 48, No. 4, 451, 1998

[4] JELLISON, G. E.: Thin Solid Films, 313 - 314, 33, 1998

[5] NÁDAŽDY, V, DURNÝ, R., THURZO, I., PINČÍK, E., NISHIDA, A., SHIMIZU, J., KUMEDA, M., SHIMIZU, T.: Phys. Rev. B 66, 195211, 2002.

[6] MÜLlEROVÁ, J., JUREČKA, S., ŠUTTA, P.: Acta Physica Slovaca, 55, No. 3, 351, 2005. 